\title{
Nonlinear current-voltage relations in polycrystalline perovskite manganites ceramics
}

\author{
John Philip and T. R. N. Kutty ${ }^{\text {a) }}$ \\ Materials Research Centre, Indian Institute of Science, Bangalore 560 012, India
}

\begin{abstract}
Highly nonlinear current-voltage $\left(J \propto E^{\alpha}\right)$ relations with voltage-limiting characteristics are observed for Mg-doped lanthanum calcium manganite polycrystalline ceramics with nonlinearity coefficient, $\alpha=2-9$ at low-electric-field strengths of $2-5 \mathrm{~V} / \mathrm{mm}$, below magnetic transition temperatures. The current density increases with external magnetic field, so that magnetically tunable low-voltage varistors are realized. The $\alpha$ increases on annealing at $1375 \mathrm{~K}$ in atmospheres of lower $p_{\mathrm{O}_{2}}$, and becomes more pronounced with decreasing grain size, indicating that nonlinear behavior is related to the outdiffusion of oxygen through the grain-boundary-layer regions. The increasing deficiency in $\mathrm{Mn}^{3+} / \mathrm{Mn}^{4+}$ pairs reduces the hole-hopping probability leading to insulating barriers in the grain-boundary-layer regions. The barrier height is lowered by the external electric field, facilitating the charge-carrier movement between the grains. Since the conduction is due to spin-polarized tunneling, the external magnetic field increases the current.
\end{abstract}

Electrical transport and magnetic properties of perovskite manganites are greatly influenced by the grain size as well as grain-boundary-layer (GBL) contributions. There is a larger drop in resistivity observed at low magnetic fields in polycrystalline specimens of $\mathrm{La}_{0.67} \mathrm{Sr}_{0.33} \mathrm{MnO}_{3}$ than that of single crystals, and this is attributed to the spin-polarized tunneling of electrons between the grains. ${ }^{1}$ There are also studies in thin films of $\mathrm{La}_{0.67}(\mathrm{Ca} / \mathrm{Sr} / \mathrm{Ba})_{0.33} \mathrm{MnO}_{3}$ with different grain sizes, compared with the properties of epitaxial films. ${ }^{2}$ Nonlinear electrical conductivity below magnetic transition temperature $\left(T_{c}\right)$ is observed in $\mathrm{La}_{0.67}(\mathrm{Ca} /$ $\mathrm{Sr})_{0.33} \mathrm{MnO}_{3}$ deposited on $\mathrm{SrTiO}_{3}$ bicrystal substrates. ${ }^{3,4}$ The nonlinear conduction is explained in terms of the presence of disordered, few-nm-wide, paramagnetic grain-boundary layer that is depleted below $T_{c} \cdot{ }^{3}$ Since perovskite manganites are potential candidates for sensor applications; the device optimizations require in-depth understanding of the microstructure effects on electrical transport as well as magnetic properties. We have prepared $\mathrm{La}_{0.67} \mathrm{Ca}_{0.33} \mathrm{Mn}_{0.95} \mathrm{Mg}_{0.05} \mathrm{O}_{3}$ (LCMMO) specimens with large difference in grain sizes, which are ideal for studying the effects of grain size and the influence of the GBL regions in manganites. LCMMO specimens annealed in atmospheres of lower $p_{\mathrm{O}_{2}}\left(\sim 10^{-6} \mathrm{~atm}\right)$ exhibit nonlinear current-voltage ( $J-E$ ) characteristics below $T_{c}$. By controlling the outdiffusion of oxygen through the GBL regions, nonlinear behavior is observed at relatively low-electric-field strengths $(<5 \mathrm{~V} / \mathrm{mm})$ with nonlinearity coefficient $\alpha=2-9$. The voltage-limiting nonlinear conduction in LCMMO can be utilized in low-voltage magnetically tunable surge protection devices (varistors), electromagnetic switching devices, and fabrication of electromagnets on the micrometer scale.

LCMMO compounds were prepared through a wetchemical method involving a redox reaction. The details of

${ }^{a)}$ Electronic mail: kutty@mrc.iisc.ernet.in the method were given in our previous publications. ${ }^{5,6}$ The phase-pure powders were pressed into disks and sintered in air at 1575 (A samples) or $1825 \mathrm{~K}$ (B samples). The durations of sintering ranged from $4-8 \mathrm{~h}$. The excess or deficiency of oxygen $(\delta)$ in $\mathrm{La}_{0.67} \mathrm{Ca}_{33} \mathrm{Mn}_{0.95} \mathrm{M}_{0.05} \mathrm{O}_{3 \pm \delta}$ was determined by iodometric titrations. Both sets of samples exhibit oxygen excess stoichiometry and can be represented as $\mathrm{La}_{0.67} \mathrm{Ca}_{0.33} \mathrm{Mn}_{0.95} \mathrm{M}_{0.05} \mathrm{O}_{3+\delta}$. The $\delta$ values are presented in Table I. X-ray diffraction patterns were obtained using a Scintag diffractometer, which indicated cubic symmetry in these specimens. The $T_{c}$ values were obtained from the dc susceptibility measurements ${ }^{7}$ and are presented in Table I. Electrical resistivity $\rho(\mathrm{T})$ was measured using the four-probe dc technique. The $J-E$ characteristics were measured using a circuit consisting of a load resistor and the sample in series. Pulse voltages with $2 \mathrm{~ms}$ duration were applied and the current in the circuit was measured with a multichannel storage oscilloscope. During the $J-E$ measurements, the body temperatures of the specimens were measured with a carbon resistor thermometer, which was made electrically insulating whereas thermally conductive, using beryllia cement. Microstructural studies were done using a S360 Cambridge scanning electron microscope (SEM).

Figure 1 shows the SEM micrographs of specimens from the $\mathrm{A}$ and $\mathrm{B}$ series. The $\mathrm{A}$ samples show an average grain

TABLE I. $\delta$ and $\alpha$ values at two different temperatures at chosen electricfield strengths and $T_{c}$ of $\mathrm{La}_{0.67} \mathrm{Ca}_{0.33} \mathrm{Mn}_{0.95} \mathrm{Mg}_{0.05} \mathrm{O}_{3}$ samples with two different sizes.

\begin{tabular}{cccccc}
\hline \hline $\begin{array}{c}\text { Temperature of } \\
\text { sintering (K) }\end{array}$ & $\delta$ & $\begin{array}{c}\text { Temperature of } \\
\text { measurement }(\mathrm{K}) \\
(J-E \text { curves })\end{array}$ & $\alpha$ & $\begin{array}{c}\text { Field strength } \\
(\mathrm{V} / \mathrm{cm})\end{array}$ & $T_{c}(\mathrm{~K})$ \\
\hline 1825 & 0.026 & 193 & 1.6 & 25 & \\
& & 150 & 3 & 37 & 206 \\
1575 & 0.028 & 193 & 2 & 120 & \\
& & 150 & 7 & 220 & \\
\hline \hline
\end{tabular}



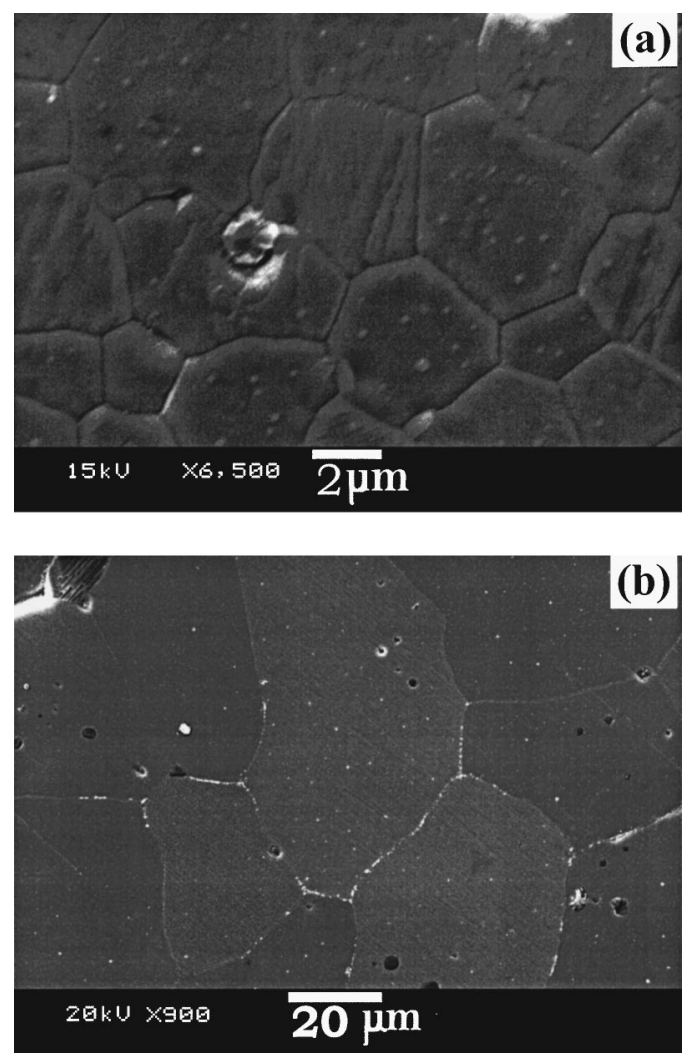

FIG. 1. SEM micrographs of $\mathrm{La}_{0.67} \mathrm{Ca}_{0.33} \mathrm{Mn}_{0.95} \mathrm{Mg}_{0.05} \mathrm{O}_{3+\delta}$ samples sintered at (a) $1575 \mathrm{~K}$ for $4 \mathrm{~h}$ and (b) $1825 \mathrm{~K}$ for $6 \mathrm{~h}$. The white particles seen in the micrograph are alumina (retained even after sonification), used for polishing.

size of $4 \mu \mathrm{m}$. The B samples display large grain growth with an average grain size of $60 \mu \mathrm{m}$. Both sets of samples are very dense, except for a few random pores.

Figure 2 displays the $\rho(T)$ behavior of the A and B series of the samples. Both these sets of samples (as sintered) show a metal-insulator (MI) transition. The larger-grainsized specimens have lower resistivity. As the grain size increases, the grain-boundary contributions to the resistivity are minimized, exhibiting larger conductivity. ${ }^{2}$ There is a shoulder peak observed at lower temperatures in the B samples. This arises due to the oxygen inhomogeneity in the samples. Oxygen-annealed samples do not show any such peaks [Fig. 2(b)]. Both sets of as-sintered specimens were annealed in atmospheres of lower $p_{\mathrm{O}_{2}}\left(10^{-6} \mathrm{~atm}\right.$, $\mathrm{CO} / \mathrm{CO}_{2}+$ Ar gas mixtures) at $1375 \mathrm{~K}$ for $1 \mathrm{~h}$. A remarkable increase in resistivity is observed in the A samples. The $\delta$ value is reduced from +0.028 to -0.06 . The MI transition is obliterated and the resistivity increases by two orders of magnitude at low temperatures. The B samples $(\delta=-0.02)$ do not display any prominent changes in resistivity. There is a cusp observed near $150 \mathrm{~K}$ where the as-sintered specimens display the MI transition; however, the overall behavior is insulating. Samples heat treated at lower $p_{\mathrm{O}_{2}}$ are reannealed in $\mathrm{O}_{2}$ atmosphere at $1375 \mathrm{~K}$ for shorter durations (10-30 min). The A samples show one order decrease in $\rho$ together with the reappearance of the MI transition. Oxygen-annealed B samples also exhibit the MI transition, but the range of resistivity values is unchanged. Thus, large variations in resistivity are observed only in A-samples after annealing in different $p_{\mathrm{O}_{2}}$. Reannealing the samples for shorter duration
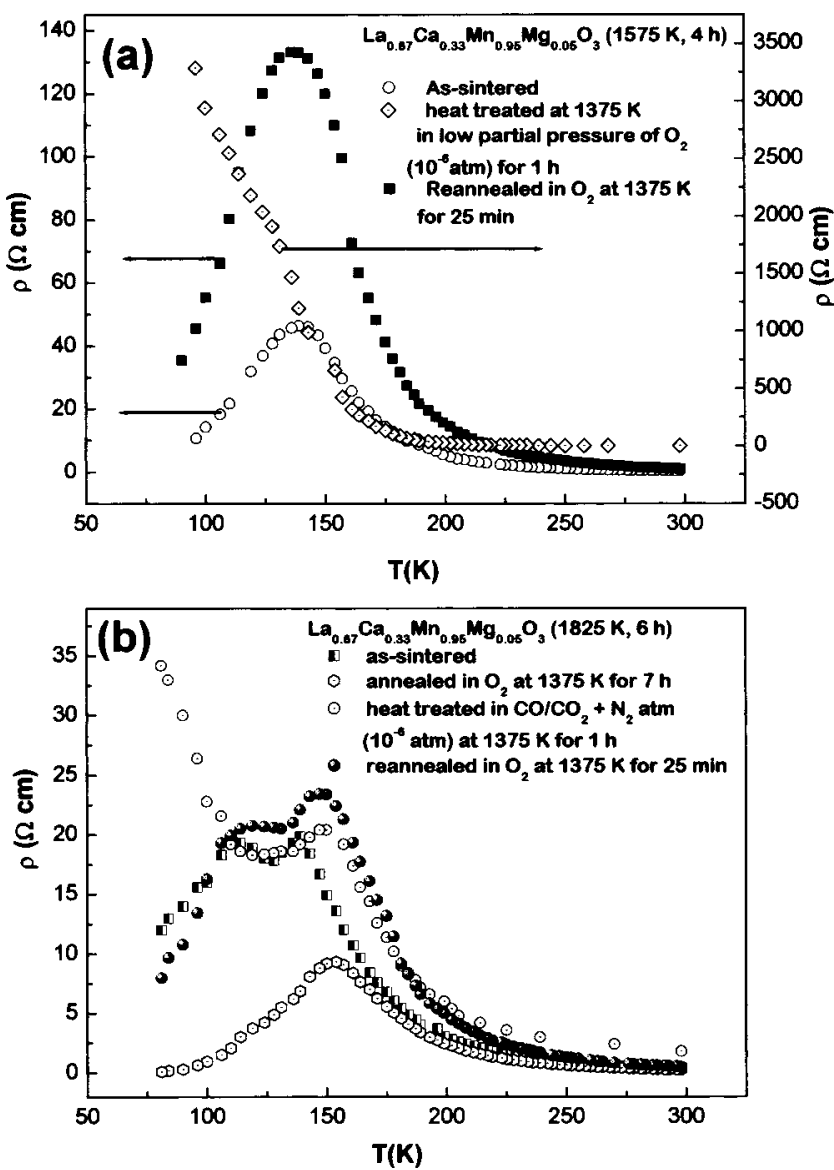

FIG. 2. Resistivity characteristics of (a) A samples and (b) B samples annealed at $1375 \mathrm{~K}$ in different $p_{\mathrm{O}_{2}}$ after sintering.

was intended to study the effect of the GBL regions on oxygen diffusion. It is well known that the diffusion of constituents around the grain boundaries is much faster than that through grain interiors. Thus, diffusion of oxygen through the bulk grain requires a longer duration of annealing. The A samples have a larger area covered by the GBL regions, permitting higher amounts of oxygen to diffuse in or out. In the case of the B samples, the grains cover a larger area and the diffusion of oxygen through the grain interiors is slower; therefore, conspicuous changes are not observed. Since the A samples take up more oxygen in the GBL regions, the $\mathrm{Mn}^{4+}$ content is also enhanced around the same regions.

Figure 3 shows the $J-E$ behavior of specimens from the A and $\mathrm{B}$ series annealed at low $p_{\mathrm{O}_{2}}\left(10^{-6} \mathrm{~atm}\right)$ at $1375 \mathrm{~K}$ for $1 \mathrm{~h}$. These specimens show insulating behavior. The curves corresponding to the filled points in Fig. 3 are the $J-E$ curves of the B samples. At higher temperatures, both sets of samples show linear conduction behavior, however, below $T_{c}$ nonlinear behavior is observed at higher-electric-field strengths. The nonlinear behavior increases inversely with temperature and can be calculated using the parameter $\alpha$ defined as $d \ln J / d \ln E$. If the non-Ohmic behavior observed arises due to Joule heating, we should have observed nonlinear $J-E$ curves even at higher temperatures where the current density is much larger than what prevails at low temperatures. Furthermore, the body temperature of the samples does not increase more than $2^{\circ}$ from that of the ambient.

There exists considerable differences in $J-E$ curves of the samples of the A and B series after heat treatment in 


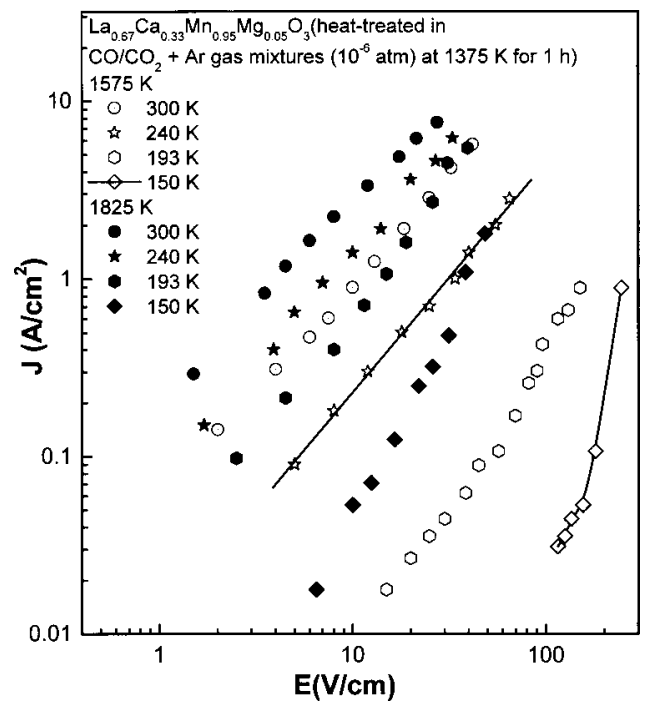

FIG. 3. $J-E$ curves of $\mathrm{A}$ and $\mathrm{B}$ samples heat treated in lower $p_{\mathrm{O}_{2}}$ $\left(10^{-6} \mathrm{~atm}\right)$ at $1375 \mathrm{~K}$ for $1 \mathrm{~h}$.

lower $p_{\mathrm{O}_{2}}$; similar to that of observed in the $\rho(T)$ curves. Below $T_{c}$, the A samples show highly nonlinear $J-E$ curves at high-field strengths in comparison to the B samples. The $\alpha$ values are calculated in the nonlinear region at chosen field strengths for different temperatures (Table I). The extent of the nonlinearity observed in the B samples is substantially small. The difference in $\alpha$ values between the A and $\mathrm{B}$ samples increases inversely with temperature. The A samples showed discernible outdiffusion of oxygen and the extent of nonlinearity is higher. The extent of oxygen diffusion in the B samples is less and the nonlinearity is correspondingly small. By annealing at different temperatures and durations in the same $p_{\mathrm{O}_{2}}$, the extent of oxygen diffusion can be controlled. Figure 4 shows the $J-E$ characteristics of the A series annealed in lower $p_{\mathrm{O}_{2}}\left(10^{-6} \mathrm{~atm}\right)$ at $1275 \mathrm{~K}$ for $50 \mathrm{~min}$. The $\delta$ value reduces from +0.028 to -0.043 . The nonlinear

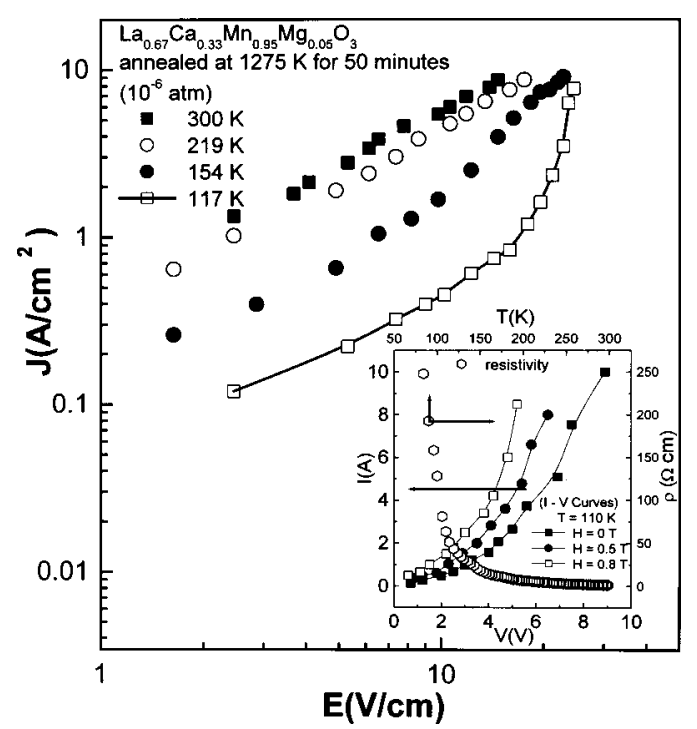

FIG. 4. $J-E$ characteristics of the A samples annealed in lower $p_{\mathrm{O}_{2}}$ $\left(10^{-6} \mathrm{~atm}\right)$ at $1275 \mathrm{~K}$ for $50 \mathrm{~min}$. The inset shows the resistivity and $J-E$ curves in the presence of different magnetic field at $T=110 \mathrm{~K}$. behavior is observed at lower-electric-field strengths $(<5 \mathrm{~V} / \mathrm{mm})$ below $T_{c}$. The nonlinearity coefficient $\alpha$ increases from $2(154 \mathrm{~K}, E=1.6 \mathrm{~V} / \mathrm{mm})$ to $9(117 \mathrm{~K}, E$ $=2.3 \mathrm{~V} / \mathrm{mm})$. The inset shows the $\rho(\mathrm{T})$ behavior; the resistivity has decreased in comparison to the same sample annealed at $1375 \mathrm{~K}$ for $1 \mathrm{~h}\left(10^{-6} \mathrm{~atm}\right)$. Thus, depending upon $p_{\mathrm{O}_{2}}$ of the annealing and the grain size, the nonlinear conduction characteristics can be tailored. The inset also shows the magnetic-field effect on the $J-E$ curves. The current in the nonlinear region increases with external magnetic field and the nonlinear region sets in at lower voltages. Thus, the magnetic field can tune the $J-E$ characteristics at which the nonlinear conduction prevails and can be used as magnetically tunable low voltage surge protection devices. There are several other low-voltage varistors reported in the literature based on $n$-type materials such as $\mathrm{ZnO},{ }^{8} \mathrm{Zn}_{7} \mathrm{Sb}_{2} \mathrm{O}_{12},{ }^{9}$ or $\mathrm{SrTiO}_{3} .{ }^{10}$ The present material differs from all of them from the fact that it is $p$ type, nonlinearity is observed below $T_{c}$, and the $J-E$ characteristics are magnetically tunable.

Only oxygen-deficient LCMMO specimens exhibit the insulating resistivity characteristics and nonlinear conductivity. Higher concentrations of oxygen vacancies around the GBL arise as a result of the outdiffusion of oxygen from the grain boundaries. Consequently, there will be a low concentration of $\mathrm{Mn}^{4+}$ ions around these regions. This results in a smaller number of $\mathrm{Mn}^{3+}-\mathrm{O}-\mathrm{Mn}^{4+}$ pairs, thereby reducing the probability of the hole hopping that responsible for conduction, leading to the insulating behavior in the GBL regions. Thus, the grain interiors are more conductive than the grain-boundary regions. Since the $\mathrm{Mn}^{4+}$ ion concentration in $\mathrm{La}_{0.67} \mathrm{Ca}_{0.33} \mathrm{Mn}_{0.95} \mathrm{Mg}_{0.05} \mathrm{O}_{3-\delta}$ is lower around the grain boundaries, the ferromagnetic transition temperature in the GBL regions [designated as $T_{c}(\mathrm{gb})$ ] will be lower in comparison to that of the grain interior (designated as $T_{c}$ ). Below $T_{c}$, the grain interiors become ferromagnetic, whereas the GBL regions stay paramagnetic due to the fact that $T_{c}(\mathrm{gb})$ $<T_{c}$. Thus, there will be band-bending effects as envisaged by Gross et al., ${ }^{3}$ giving rise to the depletion regions. The nonlinear $J-E$ curves arise from the insulating barrier in the GBL regions. Below $T_{c}$, the conduction across the barrier may be due to the spin-dependent tunneling. When the barrier height is lowered by the application of an external electric field, energetic carriers can move over the barrier, resulting in larger conductivity. When the applied electric field reaches a threshold value, the barrier height is remarkably lowered, allowing a large number of carriers to move between the grains, and thereby exhibiting a higher current density.

${ }^{1}$ H. Y. Hwang, S-W. Chong, N. P. Ong, and B. Batlog, Phys. Rev. Lett. 77, 2041 (1996).

${ }^{2}$ A. Gupta, G. Q. Gong, Gang Xiao, et al., Phys. Rev. B 54, R15629 (1996).

${ }^{3}$ R. Gross, L. Alff, B. Buchner, et al., J. Magn. Magn. Mater. 211, 150 (2000).

${ }^{4}$ N. K. Todd, N. D. Mathur, S. P. Issac, J. E. Evetts, and M. G. Blamire, J. Appl. Phys. 85, 7263 (1999).

${ }^{5}$ J. Philip and T. R. N. Kutty, Mater. Phys. Chem. 63, 218 (2000).

${ }^{6}$ J. Philip and T. R. N. Kutty, J. Phys.: Condens. Matter 11, 8537 (1999).

${ }^{7}$ J. Crangle, The Magnetic Properties of Solids (Arnold, London, 1977).

${ }^{8}$ T. R. N. Kutty and S. Ezhilvalavan, Mater. Lett. 27, 355 (1996).

${ }^{9}$ S. Ezhilvalavan and T. R. N. Kutty, Appl. Phys. Lett. 68, 2693 (1996).

${ }^{10}$ T. R. N. Kutty and Sam Philip, Mater. Sci. Eng., B 33, 58 (1995). 